OPEN ACCESS

Edited by:

Phyo Kyaw Myint, University of Aberdeen,

United Kingdom

Reviewed by:

Anita Ante Arsovska, Saints Cyril and Methodius University

of Skopje, Macedonia

Steve Simpson-Yap,

The University of Melbourne, Australia

*Correspondence:

Yu Wang

yw4d@hotmail.com

Jun-Cang Wu

wujuncang126@126.com

Specialty section:

This article was submitted to

Stroke,

a section of the journa

Frontiers in Neurology

Received: 05 April 2021 Accepted: 30 September 2021 Published: 05 November 2021

Citation:

Wang L, Zhao X-m, Wang F-y, Wu J-C and Wang $Y$ (2021) Effect of Vitamin D Supplementation on the Prognosis of Post-stroke Fatigue: A Retrospective

Cohort Study.

Front. Neurol. 12:690969

doi: 10.3389/fneur.2021.690969

\section{Effect of Vitamin D Supplementation on the Prognosis of Post-stroke Fatigue: A Retrospective Cohort Study}

\author{
Long Wang ${ }^{1,2}$, Xue-min Zhao ${ }^{2}$, Fu-yu Wang ${ }^{3}$, Jun-Cang Wu ${ }^{1 *}$ and Yu Wang ${ }^{4 *}$ \\ ' Department of Neurology, The Second People's Hospital of Hefei, Hefei, China, ${ }^{2}$ Department of Neurology, General Hospital \\ of Wan Bei Coal and Electrical Group, Suzhou, China, ${ }^{3}$ Department of Pharmacy, The Second People's Hospital of Hefei, \\ Hefei, China, ${ }^{4}$ Department of Neurology, The First Affiliated Hospital of Anhui Medical University, Hefei, China
}

Objective: We aimed to evaluate the effect of vitamin D supplementation in post-stroke fatigue (PSF) patients with vitamin D deficiency on fatigue symptoms and outcomes.

Methods: Patients with primary acute ischemic stroke (AIS) were recruited consecutively from July 2016 to June 2018. Post-stroke fatigue patients were screened out with the Fatigue Severity Scale (FSS) questionnaire, serum concentrations of 25-hydroxyvitamin D [25-(OH)-D] were assessed with enzyme-linked immunosorbent assay (ELISA), and neurological function was evaluated with FSS and modified Rankin Scale (mRS) scoring criteria. Post-stroke fatigue patients with vitamin D deficiency were divided into two groups: a study group in which patients received vitamin $D$ supplementation (cholecalciferol, $600 \mathrm{IU} /$ day) along with usual care, and a control group in which patients received usual care alone. At the end of 1 and 3 months after treatment, all PSE patients accepted re-measurement of serum vitamin $D$ and re-evaluation of fatigue and neurological function.

Results: A total of 532 AIS patients were consecutively recruited to participate in this study. Patients without PSF, non-vitamin D deficiency, pre-stroke fatigue, or vitamin D supplementation were excluded from the study. In addition, patients who were lost to follow-up were also excluded. Finally, 139 out of 532 (26.1\%) patients with PSF and vitamin $\mathrm{D}$ deficiency received vitamin $\mathrm{D}$ supplementation treatment. Fatigue Severity Scale score was significantly lower in the study group than in the control group at 1 month $(t=-4.731, p<0.01)$ and 3 months $(t=-7.937, p<0.01)$ after treatment. One month after treatment, mRS score in the study group was lower than that in the control group without statistical difference $(t=-0.660, p>0.05)$, whereas mRS was significantly higher in the study group than in the control group at 3 months after treatment $(t=-4.715, p<0.01)$.

Conclusions: Our results indicated that vitamin D supplementation could improve fatigue symptoms and neurological outcomes in PSF patients with vitamin D deficiency. Subject to replication in other settings, a randomized controlled trial (RCT) might be undertaken to validate the potential beneficial impact of vitamin D supplementation in post-stroke patients found to be vitamin $\mathrm{D}$ deficient.

Keywords: post-stroke, fatigue, vitamin D, supplementation, outcome 


\section{INTRODUCTION}

Post-stroke fatigue (PSF) is often overlooked though it is common in patients after stroke. The estimated prevalence of PSF ranges from 23 to $75 \%$, and its symptoms may continue up to 1 year or more (1-3). Post-stroke fatigue has been defined as pathological tiredness unrelated to intensity of exercise in the post-stroke period. Apart from distress reported by PSD patients, PSF has been found to be related to the increase of mortality (4). Although the etiology of PSF remains unclear, it is reported that the occurrence of PSF is associated with several predisposing factors such as vitamin D deficiency, depression, and premorbid fatigue (5-7).

In humans, vitamin $\mathrm{D}$, a fat-soluble hormone, is supplied from different sources such as skin exposure to light (8), food, or by supplementation. Vitamin D is converted to 25hydroxyvitamin D [25-(OH)-D] in the liver by different 25hydroxylase enzymes. Measurement of serum level of 25-(OH)-D is used to reflect the level of vitamin D as it best reflects vitamin D supply from all different sources (9). In recent years, studies have found that vitamin D level not only plays a role in regulating calcium and phosphorus metabolism but also has association with the severity and prognosis of stroke (10-12). Vitamin D has a protective effect on endothelial function, vascular remodeling, and neuromuscular and immunological functions in experimental models $(13,14)$. Additionally, recent lines of evidence have shown a close link between vitamin D deficiency and fatigue occurrence in both healthy and clinical conditions (15-17).

One meta-analysis had shown that vitamin D deficiency was related with 2.5-fold increase of the risk of acute ischemic stroke (AIS) (18). Another meta-analysis involving 28,072 patients suggested that calcium supplementation, with or without vitamin $\mathrm{D}$ deficiency, could modestly increase the risk of stroke (19). It is noteworthy that these studies were aimed at primary prevention of stroke but not at post-stroke conditions. The benefit of vitamin D supplementation in improving outcomes in AIS patients remains controversial $(20,21)$.

Considering the involvement of vitamin $\mathrm{D}$ in fatigue and outcomes in AIS, we retrospectively analyzed the efficacy of vitamin $\mathrm{D}$ supplementation in fatigue and neurological outcome improvement in PSF patients with vitamin D deficiency through 3 months' follow-up.

\section{METHODS}

\section{Study Design}

We conducted a retrospective study to assess the effectiveness of vitamin D supplementation on the prognosis of PSF in stroke patients admitted to the Department of Neurology, General Hospital of Wan Bei Coal and Electrical Group from July 2016 to June 2018.

This study was approved by the General Hospital of Wan Bei Coal and Electrical Group Research Ethics Board and without the need for informed consent by using the medical data of the participants.

\section{Inclusion and Exclusion Criteria}

Fatigue was examined using the Fatigue Severity Scale (FSS), which was proved to be valid and reliable in various clinical groups $(22,23)$. The FSS contains seven items where higher mean scores reflect higher degrees of fatigue (24). Fatigue was defined as mean FSS score $\geq 4$ (23).

All enrolled patients with AIS were confirmed by $3.0 \mathrm{~T}$ cranial magnetic resonance imaging (MRI) with the mean FSS scored above four points. Inclusion criteria were as follows: (1) aged between 40 and 75 years old; (2) first-ever stroke with MRI scan upon admission and confirmed AIS; and (3) FSS score was evaluated at 1 week after onset.

The exclusion criteria were as follows: (1) diagnosed with transient ischemic attack; (2) previous stroke or any other central nervous system disease, such as brain trauma, tumor, and cerebritis; (3) fatigue within 1 month before admission; (4) severe liver and kidney dysfunction; (5) taking vitamin D or other drugs affecting bone metabolism within 3 months; and (6) cognitive or speech disorders that may affect evaluation.

\section{Data Collection}

The patients' demographic characteristics including gender and age were obtained from their medical records. Baseline clinical parameters were collected, including stroke subtype (based on the TOAST criteria), current smoking, and arterial blood pressure. Pre-existing comorbidities included hypertension, diabetes, and coronary heart disease (CHD). The baseline laboratory tests, including 25-(OH)-D, fasting blood glucose (FBG), low-density lipoprotein cholesterol (LDL-C), total cholesterol (TC), and uric acid (UA), were obtained within $24 \mathrm{~h}$ of admission. Additionally, the stroke severity was assessed by experienced neurologists according to the NIHSS within $24 \mathrm{~h}$ of admission.

\section{Serum 25-(OH)-D Assessment and Treatment}

Venous blood samples were collected in plain biochemical tube within $24 \mathrm{~h}$ of admission. Serum 25-(OH)-D concentration was measured by chemiluminescent immunoassay (Cobase 6000, Roche Diagnostics, Swiss). Similar to previous studies, a normal serum level of vitamin D was defined as $25-(\mathrm{OH})-\mathrm{D}$ concentration $>30 \mathrm{ng} / \mathrm{ml}(25)$. Vitamin D deficiency was defined as $25-(\mathrm{OH})$-D concentration $<20 \mathrm{ng} / \mathrm{ml}(25,26)$.

Study group: patients with vitamin D deficiency in the study were treated with combination therapy of vitamin $\mathrm{D}_{3}$ ( (cholecalciferol, $600 \mathrm{IU} /$ day) for 3 months.

Control group: patients with vitamin D deficiency were not treated with combined vitamin $\mathrm{D}$ due to out-of-pocket payment or lack of cooperation.

In addition, all enrolled patients were treated in accordance with the American Heart/Stroke Association Guidelines for the Management of Acute Ischemic Stroke (2018) (27). Individual treatment was planned based on the condition of each patient.

\section{Follow-Up Records}

The $\mathrm{mRS}$ is a graded interval scale [range, 0 (no symptoms) to 6 (death)] for the assessment of neurological function (28). 


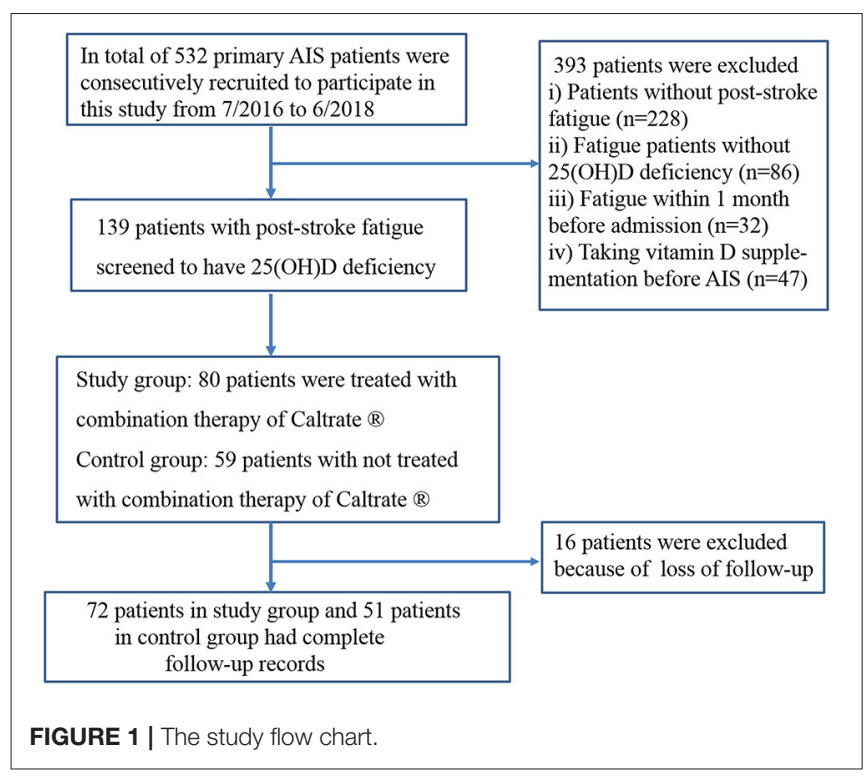

Serum 25-(OH)-D levels, FSS, and mRS scores were obtained from their follow-up records with re-measurement and reevaluation at 1 and 3 months after treatment.

\section{Statistical Analysis}

Continuous variables were described as mean ( \pm standard deviation) for normally distributed data and median (four percentile) (25th and 75th interquartile range) for nonnormally distributed data. Categorical variables were presented as numbers (percentages). The paired $t$-test for two means (paired observations) were applied for analyzing within-group changes from baseline in quantitative parameters. The repeated measure test was applied for analyzing the differences in categorical parameters between the study groups. Kruskal-Wallis test was used for statistical analysis of non-normal distribution data among groups. Pearson's correlations $(r)$ were conducted to analyze the relationship between FSS scores and 25-(OH)-D levels. All analyses were carried out using SPSS (version 17.0). A $p$-value $<0.05$ was considered to be statistically significant.

\section{RESULTS}

\section{Study Process}

As illustrated in the study flow in Figure 1, a total of 139 patients met all inclusion criteria and screened to have vitamin $\mathrm{D}$ deficiency. Seventy-two patients in the study group and 51 patients in the control group had complete follow-up records (Figure 1).

\section{Baseline Characteristics of Patients}

Two groups did not differ in respect to baseline characteristics, including age, gender, clinical parameters, pre-existing comorbidities, and biochemical variables. The demographics and characteristics of the patients are displayed in Table $\mathbf{1}$.
TABLE 1 | The demographic and clinical aspects of the study population.

\begin{tabular}{lcccc}
\hline & $\begin{array}{c}\text { Study group } \\
(\boldsymbol{n}=\mathbf{7 2})\end{array}$ & $\begin{array}{c}\text { Control group } \\
(\boldsymbol{n}=\mathbf{5 1})\end{array}$ & $\boldsymbol{t} / \chi^{\mathbf{2}}$ & $\boldsymbol{P}$ \\
\hline Age $( \pm$ SD), years & $62.0 \pm 8.2$ & $64.4 \pm 8.6$ & -1.418 & 0.159 \\
Male, $n \%$ & $30(41.7)$ & $29(56.9)$ & 2.762 & 0.097 \\
Hypertension, $n(\%)$ & $40(55.6)$ & $32(62.7)$ & 0.636 & 0.425 \\
Diabetes, $n(\%)$ & $25(34.7)$ & $14(27.5)$ & 0.729 & 0.393 \\
CHD, $n$ (\%) & $2(2.8)$ & $3(5.9)$ & 0.738 & 0.390 \\
Hyperlipidemia, $n(\%)$ & $43(59.7)$ & $22(45.1)$ & 3.295 & 0.069 \\
Current smoking, $n(\%)$ & $33(45.8)$ & $17(33.3)$ & 1.933 & 0.164 \\
Stroke subtype, $n(\%)$ & & & 1.456 & 0.692 \\
$\quad$ LAA & $41(56.9)$ & $26(51.0)$ & & \\
SVO & $28(38.9)$ & $24(47.0)$ & & \\
CE & $2(2.8)$ & $1(2.0)$ & & \\
$\quad$ SOE and SUE & $1(1.4)$ & 0 & & \\
IV thrombolysis & $4(5.6)$ & $1(2.0)$ & 0.989 & 0.320 \\
NIHSS score & $5.8 \pm 2.5$ & $5.1 \pm 2.2$ & 1.479 & 0.142 \\
FSS score & $5.4 \pm 1.4$ & $5.4 \pm 1.2$ & 0.161 & 0.872 \\
FBG, mmol/L & $6.8 \pm 1.9$ & $6.5 \pm 2.0$ & 0.880 & 0.380 \\
$\quad$ TC, mmol/L & $4.7 \pm 1.0$ & $4.4 \pm 0.9$ & 1.827 & 0.070 \\
LDL, mmol/L & $2.8 \pm 0.9$ & $2.6 \pm 0.6$ & 1.278 & 0.204 \\
UA, mmol/L & $317.9 \pm 89.2$ & $297.0 \pm 82.1$ & 1.318 & 0.190 \\
25-(OH)-D, ng/ml & $13.1 \pm 3.6$ & $13.9 \pm 4.3$ & -1.323 & 0.188 \\
\hline & & & & \\
\hline
\end{tabular}

CHD, coronary heart disease; LAA, large artery atherosclerosis; SVO, small vessel occlusion; CE, cardioembolism; SOE, stroke of other determined etiology; SUE, stroke of undetermined etiology; NIHSS, National Institute of Health Stroke Scale; IV, intravenous; $T C$, total cholesterol; HDL, high-density lipoprotein; $L D L$, low-density lipoprotein; FBG, fasting blood glucose; UA, uric acid.

TABLE 2 | Serum 25-(OH)-D levels before and after treatment between two groups.

\begin{tabular}{lcccc}
\hline Time & Study group & Control group & $\boldsymbol{t}$ & $\boldsymbol{P}$ \\
\hline Pre-treatment & $13.1 \pm 3.6$ & $13.9 \pm 4.3$ & -1.323 & 0.188 \\
Follow-up (1 month) & $22.8 \pm 3.2$ & $14.2 \pm 4.0$ & 12.795 & $<0.001$ \\
Follow-up (3 month) & $35.7 \pm 5.2$ & $16.5 \pm 4.5$ & 21.199 & $<0.001$ \\
\hline
\end{tabular}

\section{Outcomes}

The 25-(OH)-D levels before supplement in the two groups were $13.1 \pm 3.6$ and $13.9 \pm 4.3 \mathrm{ng} / \mathrm{ml}$, respectively, with no significant difference $(p>0.05)$. At the first and third month of follow-up, the mean $25-(\mathrm{OH})-\mathrm{D}$ levels in the study group were 22.8 and $35.7 \mathrm{ng} / \mathrm{ml}$, respectively, which were significantly higher than those at the corresponding time points in the control group $(p<0.01$, Table 2; Figure 2).

The FSS scores before vitamin $\mathrm{D}_{3}$ supplementation in the two groups were $5[4,7]$ and $6[4,6] \mathrm{ng} / \mathrm{ml}$, respectively, with no significant difference $(p>0.05)$. At the first and third month of follow-up, the mean FSS scores in the study group were 4.0 and $2.0 \mathrm{ng} / \mathrm{ml}$, respectively, which was significantly lower than those at the corresponding time points in the control group $(p<0.01$, Table 3; Figure 3). 
One month after treatment, $\mathrm{mRS}$ score in the study group was lower than that in the control group without statistical difference $(p>0.05)$, whereas $\mathrm{mRS}$ was significantly higher in the study group than in the control group at 3 months after treatment $(p<0.01$, Table 3; Figure 4).

Correlation analysis showed that FSS scores were negatively correlated with serum 25-(OH)-D levels $(r=-0.65, p<0.001)$.

\section{DISCUSSION}

Previous studies have demonstrated that a substantial proportion of stroke patients are afflicted with vitamin D deficiency associated with AIS severity, PSF, and worse functional outcomes (29-32). However, there are few trial studies evidencing whether vitamin $\mathrm{D}$ supplementation has a positive effect on the stroke outcome $(11,33)$.

To the best of our knowledge, the current study reports, for the first time, a dynamic evaluation of the effect of vitamin D supplementation on PSF and outcomes. After 1 month of vitamin D supplementation, the fatigue scores in the study group were significantly lower than those of the control group. Nevertheless, we did not find a difference in mRS scores between the two groups, but observed a trend toward decrease in mRS. With 25-(OH)-D level increased, a significant difference in mRS scores was observed between two groups with better

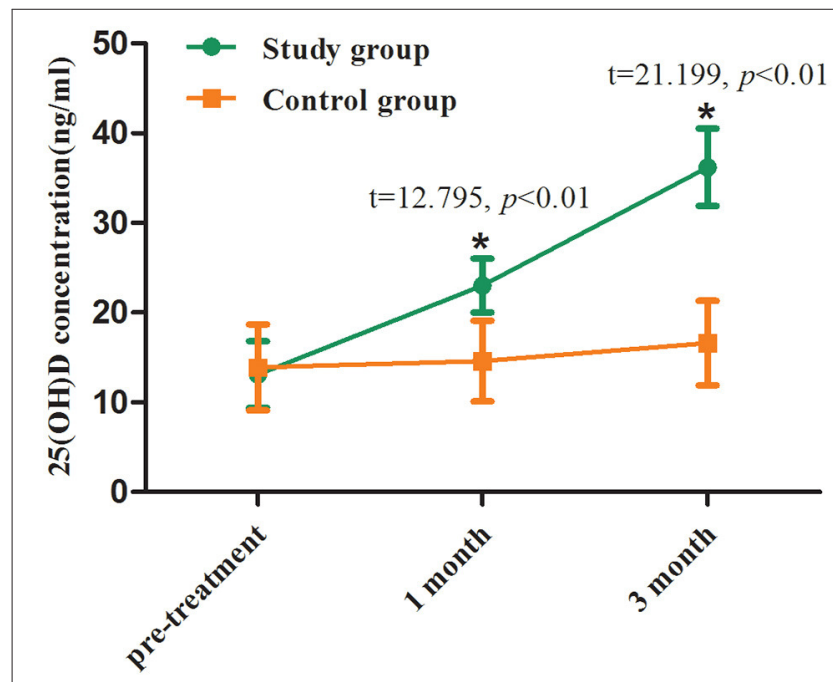

FIGURE 2 | Serum 25-(OH)-D levels before and after treatment between two groups. Comparing to the control group, ${ }^{\star} p<0.01$. neurological outcome achieved in the study group at 3 months after supplementation. Our finding may provide a potential proposal for the treatment of fatigue and functional deficits in AIS.

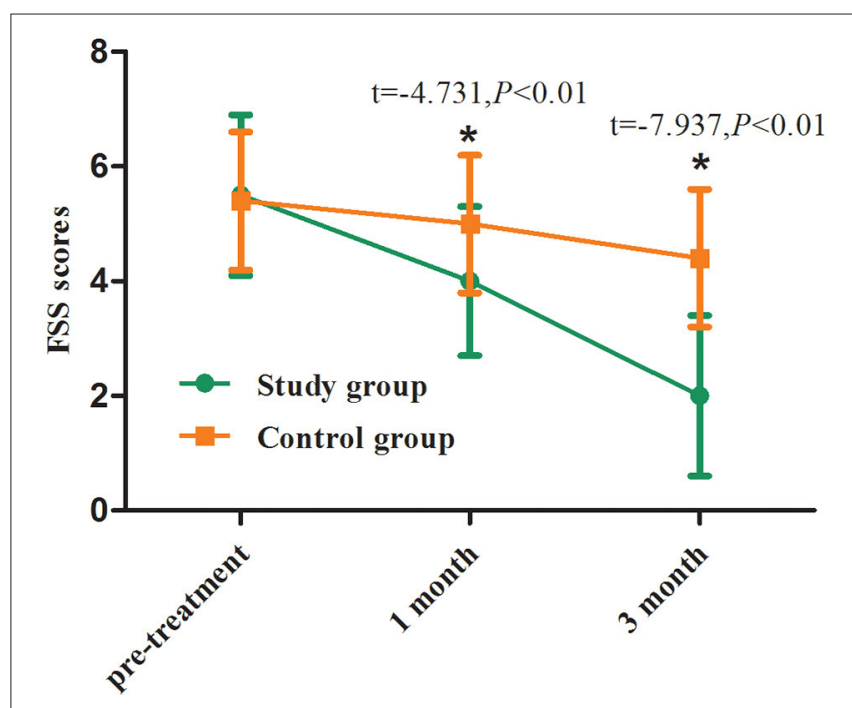

FIGURE 3 | FSS scores before and after treatment between two groups. Comparing to the control group, ${ }^{*} p<0.01$.

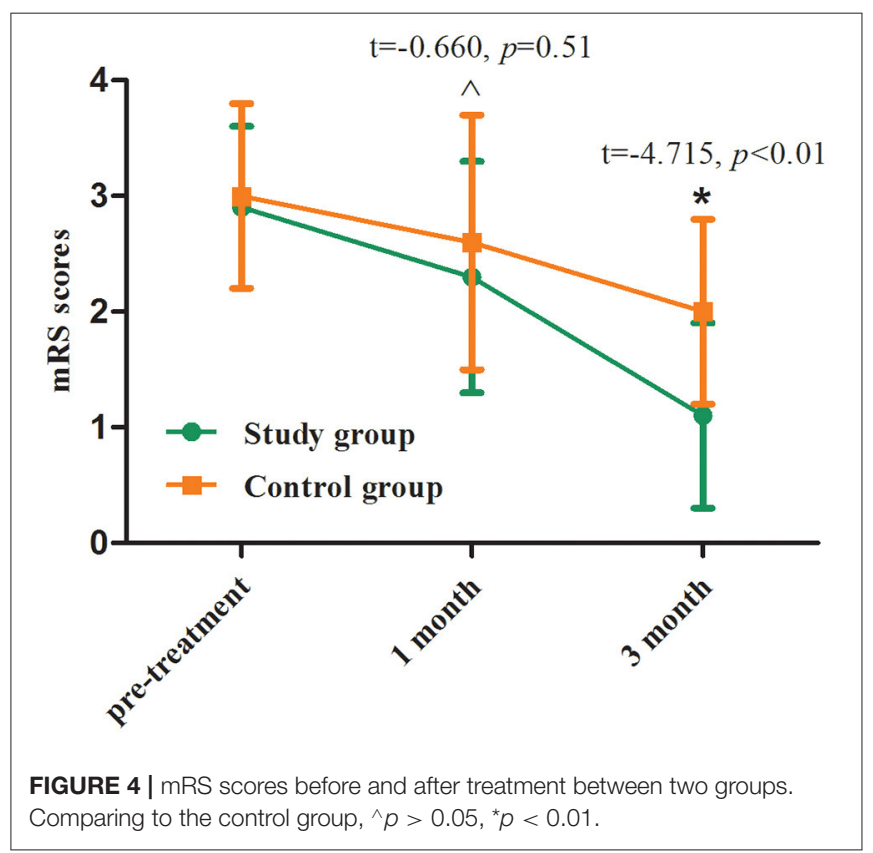

TABLE 3 | Neurological symptom scoring before and after treatment between two groups.

\begin{tabular}{|c|c|c|c|c|c|c|}
\hline \multirow[t]{2}{*}{ Variable } & \multicolumn{3}{|c|}{ Study group } & \multicolumn{3}{|c|}{ Control group } \\
\hline & Pre-treatment & Follow-up (1month) & Follow-up (3month) & Pre-treatment & Follow-up (1month) & Follow-up (3month) \\
\hline FSS, scores & $5[4,7]$ & $4[3,5]$ & $2[1,3]$ & $6[4,6]$ & $5[4,6]$ & $4[3,5]$ \\
\hline mRS, scores & $3[2,3]$ & $2[1,3]$ & $1[1,2]$ & $3[3,3]$ & $2[2,3]$ & $2[1,2]$ \\
\hline
\end{tabular}


The prospective non-randomized studies have shown that normalization of vitamin $\mathrm{D}$ concentration could improve fatigue in patients with various medical conditions (34-36). Our findings are in keeping with a recent randomized controlled trial (RCT) showing that a single intramuscular injection of vitamin $\mathrm{D}_{3}$ (600,000 IU) improved Scandinavian Stroke Scale (SSS) score in vitamin D-deficient patients at 12 weeks following AIS (21). However, another recent RCT suggested that oral vitamin D supplementation (2,000 IU/day) does not improve rehabilitation outcomes after AIS (37). These research discrepancies may be caused by a variety of mixed factors, such as supplemental dosage and mode of vitamin $\mathrm{D}$, environmental and social factors for the study subjects, study design, and outcome measures in each trials.

The mechanisms of vitamin D against PSF remain unclear to date, but several neuroprotective mechanisms have been proposed. Vitamin D deficiency induces muscle weakness and myalgia especially in stroke patients with neurological impairment $(38,39)$. Adequate vitamin D level promotes the expression of insulin-like growth factor 1, which has neuroprotective ability against axonal and dendrite degeneration $(39,40)$, as well as antithrombotic capabilities by activating plasminogen (41). Another possible explanation is that vitamin $\mathrm{D}$ is involved in inhibiting inflammation and oxidative stress, increasing post-stroke blood flow to central nervous system through enhancing the activity of nitric oxide synthase $(42,43)$. Furthermore, vitamin D therapy improves muscle mitochondrial oxidative phosphorylation that potentially causes a modulation of fatigue (44).

Previously tried drug interventions including the use of Fluoxetine and Modafinil have been shown largely ineffective for PSF $(45,46)$. Future longitudinal RCT with a larger sample size confirming our findings may provide a novel therapeutic option to improve the prognosis of PSF.

Several limitations of this study should be considered. Firstly, the limited number of subjects in our study reduced the statistical power. Secondly, other confounders such as nutritional habits and lifestyle that may affect serum level of 25-(OH)-D (47) were not assessed in this study. Thirdly, the main shortcoming of this intervention study was the lack of a placebo-control group as it was a retrospective study. Thus, a minimum replication study or, more ideally, a prospective cohort study is needed

\section{REFERENCES}

1. Cumming TB, Packer M, Kramer SF, English C. The prevalence of fatigue after stroke: a systematic review and meta-analysis. Int J Stroke. (2016) 11:968-77. doi: 10.1177/1747493016669861

2. Aarnes R, Stubberud J, Lerdal A. A literature review of factors associated with fatigue after stroke and a proposal for a framework for clinical utility. Neuropsychol Rehabil. (2019) 30:1449-76. doi: 10.1080/09602011.2019.1589530

3. Njoku K, Iveson E. Does vitamin D deficiency predispose to post stroke fatigue? Ir Med J. (2020) 113:23.

4. Naess H, Nyland H. Poststroke fatigue and depression are related to mortality in young adults: a cohort study. BMJ Open. (2013) 3:e002404. doi: 10.1136/bmjopen-2012-002404 before any RCT is considered. Despite these limitations, our findings suggested an association between vitamin $\mathrm{D}$ level and outcomes in patients with PSF and a therapeutic option may be recommended for PSF patients.

\section{CONCLUSION}

Taken together, our results indicated that vitamin D supplementation could improve fatigue symptoms and outcomes in PSF patients with vitamin D deficiency. Subject to replication in other settings, RCT might be undertaken to validate the potential beneficial impact of vitamin $\mathrm{D}$ supplementation in post-stroke patients found to be vitamin $\mathrm{D}$ deficient.

\section{DATA AVAILABILITY STATEMENT}

The original contributions presented in the study are included in the article/supplementary material, further inquiries can be directed to the corresponding author/s.

\section{ETHICS STATEMENT}

The studies involving human participants were reviewed and approved by General Hospital of Wanbei Coal and Electrical Group Research Ethics Board. Written informed consent for participation was not required for this study in accordance with the national legislation and the institutional requirements.

\section{AUTHOR CONTRIBUTIONS}

The study was conceived by LW, J-CW, and YW. Case collection and data compilation were conducted by LW and X-mZ. Follow-up was performed by F-yW, LW, and X-mZ. The rough manuscript was drafted by LW and YW. All authors reviewed and approved the final version of the manuscript.

\section{FUNDING}

This study was supported by the Beng Bu Medical College Project (2017BYKY17191) and the National Natural Science Foundation of China (81671290).
5. Wang SS, Wang JJ, Wang PX. Chen R: determinants of fatigue after first-ever ischemic stroke during acute phase. PLoS ONE. (2014) 9:e110037. doi: 10.1371/journal.pone.0110037

6. Thilarajah S, Mentiplay BF, Bower KJ, Tan D, Pua YH, Williams G, et al. Factors associated with post-stroke physical activity: a systematic review and meta-analysis. Arch Phys Med Rehabil. (2018) 99:187689. doi: 10.1016/j.apmr.2017.09.117

7. Njoku K, Iveson E. Post stroke fatigue and vitamin D deficiency. Age Ageing. (2016). 45(Suppl 1):i20. doi: 10.1093/ageing/afw034.01

8. Rajakumar K. Vitamin D, cod-liver oil, sunlight, and rickets: a historical perspective. Pediatrics. (2003) 112:e132-5. doi: 10.1542/peds.112. 2.e132

9. Bikle DD, Malmstroem S, Schwartz J. Current controversies: are free vitamin metabolite levels a more accurate assessment of vitamin $\mathrm{D}$ status 
than total levels? Endocrinol Metab Clin North Am. (2017) 46:90118. doi: 10.1016/j.ecl.2017.07.013

10. Khazai N, Judd SE, Tangpricha V. Calcium and vitamin D: skeletal and extraskeletal health. Curr Rheumatol Rep. (2008) 10:110-7. doi: 10.1007/s11926-008-0020-y

11. Kim C, Lee SH, Lim JS, Kim Y, Jang MU, Oh MS, et al. Impact of 25hydroxyvitamin $\mathrm{D}$ on the prognosis of acute ischemic stroke: machine learning approach. Front Neurol. (2020). 11:37. doi: 10.3389/fneur.2020. 00037

12. Wei L, Chen C, Dai YQ, Ding L, Li HY, Lin YJ, et al. Serum 25-hydroxyvitamin $\mathrm{D}$ deficiency predicts poor outcomes among acute ischemic stroke patients receiving intravenous thrombolysis. Chinese Med J Peking. (2019) 132:4914. doi: 10.1097/CM9.0000000000000084

13. Hamabe A, Takase B, Uehata A, Kurita A, Ohsuzu F, Tamai S. Impaired endothelium-dependent vasodilation in the brachial artery in variant angina pectoris and the effect of intravenous administration of vitamin C. Am J Cardiol. (2001) 87:1154-9. doi: 10.1016/S0002-9149(01) 01485-0

14. Al Mheid I, Patel R, Murrow J, Morris A, Rahman A, Fike L, et al. Vitamin D status is associated with arterial stiffness and vascular dysfunction in healthy humans. J Am Coll Cardiol. (2011) 58:186-92. doi: 10.1016/j.jacc.2011. 02.051

15. Pennisi M, Malaguarnera G, Di Bartolo G, Lanza G, Bella R, Chisari EM, et al. Decrease in serum vitamin D level of older patients with fatigue. Nutrients. (2019). 11:2531. doi: 10.3390/nu11102531

16. Martinez-Alonso M, Dusso A, Ariza G, Nabal M. Vitamin D deficiency and its association with fatigue and quality of life in advanced cancer patients under palliative care: a cross-sectional study. Palliat Med. (2016) 30:8996. doi: 10.1177/0269216315601954

17. Schnieders J, Willemsen D, de Boer H. Factors contributing to chronic fatigue after traumatic brain injury. J Head Trauma Rehabil. (2012) 27:40412. doi: 10.1097/HTR.0b013e3182306341

18. Zhou R, Wang MY, Huang H, Li WY, Hu YH, Wu T. Lower vitamin D status is associated with an increased risk of ischemic stroke: a systematic review and meta-analysis. Nutrients. (2018). 10:277. doi: 10.3390/nu100 30277

19. Bolland MJ, Grey A, Avenell A, Gamble GD, Reid IR. Calcium supplements with or without vitamin D and risk of cardiovascular events: reanalysis of the Women's Health Initiative limited access dataset and meta-analysis. BMJ. (2011). 342:d2040. doi: 10.1136/bmj.d2040

20. Pilz S, Tomaschitz A, Drechsler C, Zittermann A, Dekker JM, Marz W. Vitamin D supplementation: a promising approach for the prevention and treatment of strokes. Curr Drug Targets. (2011) 12:88-96. doi: 10.2174/138945011793591563

21. Gupta A, Prabhakar S, Modi M, Bhadada SK, Kalaivani M, Lal V, et al. Effect of Vitamin D and calcium supplementation on ischaemic stroke outcome: a randomised controlled open-label trial. Int J Clin Pract. (2016) 70:76470. doi: $10.1111 /$ ijcp. 12866

22. Poulsen MB, Skovbolling SL, Kruuse C, Overgaard K, Rasmussen RS. How to identify fatigue in stroke patients: an investigation of the poststroke fatigue case definition validity. Top Stroke Rehabil. (2020) 27:36976. doi: 10.1080/10749357.2019.1704387

23. Nadarajah M, Mazlan M, Abdul-Latif L, Goh HT. Test-retest reliability, internal consistency and concurrent validity of Fatigue Severity Scale in measuring post-stroke fatigue. Eur J Phys Rehabil Med. (2017) 53:7039. doi: 10.23736/S1973-9087.16.04388-4

24. Krupp LB, LaRocca NG, Muir-Nash J, Steinberg AD. The Fatigue Severity Scale. Application to patients with multiple sclerosis and systemic lupus erythematosus. Arch Neurol. (1989) 46:1121-3. doi: 10.1001/archneur.1989.00520460115022

25. Holick MF, Binkley NC, Bischoff-Ferrari HA, Gordon CM, Hanley DA, Heaney RP, et al. Evaluation, treatment, and prevention of vitamin D deficiency: an Endocrine Society clinical practice guideline. J Clin Endocrinol Metab. (2011) 96:1911-30. doi: 10.1210/jc.2011-0385

26. Ross AC, Manson JE, Abrams SA, Aloia JF, Brannon PM, Clinton SK, et al. The 2011 report on dietary reference intakes for calcium and vitamin D from the Institute of Medicine: what clinicians need to know. J Clin Endocrinol Metab. (2011). 96:53-8. doi: 10.1016/j.jada.2011.01.004
27. Powers WJ, Rabinstein AA, Ackerson T, Adeoye OM, Bambakidis NC, Becker K, et al. 2018 guidelines for the early management of patients with acute ischemic stroke: a guideline for healthcare professionals from the American Heart Association/American Stroke Association. Stroke. (2018). 49:e46-110. doi: 10.1161/STR.0000000000000211

28. Hong KS, Saver JL. Quantifying the value of stroke disability outcomes: WHO global burden of disease project disability weights for each level of the modified Rankin Scale. Stroke. (2009) 40:3828-33. doi: 10.1161/STROKEAHA.109.561365

29. Daubail B, Jacquin A, Guilland JC, Hervieu M, Osseby GV, Rouaud O, et al. Serum 25-hydroxyvitamin D predicts severity and prognosis in stroke patients. Eur J Neurol. (2013) 20:57-61. doi: 10.1111/j.1468-1331.2012. 03758.x

30. Nie Z, Ji XC, Wang J, Zhang HX. Serum levels of 25-hydroxyvitamin $\mathrm{D}$ predicts infarct volume and mortality in ischemic stroke patients. J Neuroimmunol. (2017) 313:41-5. doi: 10.1016/j.jneuroim.2017. 10.002

31. Daumas A, Daubail B, Legris N, Jacquin-Piques A, Sensenbrenner B, Denimal $\mathrm{D}$, et al. Association between admission serum 25-hydroxyvitamin D levels and functional outcome of thrombolyzed stroke patients. J Stroke Cerebrovasc. (2016) 25:907-13. doi: 10.1016/j.jstrokecerebrovasdis.2016.01.005

32. Park KY, Chung PW, Kim YB, Moon HS, Suh BC, Won YS, et al. Serum vitamin D status as a predictor of prognosis in patients with acute ischemic stroke. Cerebrovasc Dis. (2015) 40:73-80. doi: 10.1159/000434691

33. Saponaro F, Marcocci C, Zucchi R. Vitamin D status and cardiovascular outcome. J Endocrinol Invest. (2019) 42:128590. doi: 10.1007/s40618-019-01057-y

34. Khan QJ, Reddy PS, Kimler BF, Sharma P, Baxa SE, O’Dea AP, et al. Effect of vitamin $\mathrm{D}$ supplementation on serum 25 -hydroxy vitamin $\mathrm{D}$ levels, joint pain, and fatigue in women starting adjuvant letrozole treatment for breast cancer. Breast Cancer Res Treat. (2010) 119:111-8. doi: 10.1007/s10549-009-0495-x

35. Askmark H, Haggard L, Nygren I, Punga AR. Vitamin D deficiency in patients with myasthenia gravis and improvement of fatigue after supplementation of vitamin D3: a pilot study. Eur J Neurol. (2012) 19:155460. doi: 10.1111/j.1468-1331.2012.03773.x

36. Cho HJ, Hotopf M, Wessely S. The placebo response in the treatment of chronic fatigue syndrome: a systematic review and meta-analysis. Psychosom Med. (2005) 67:301-13. doi: 10.1097/01.psy.0000156969.76986.e0

37. Momosaki R, Abo M, Urashima M. Vitamin D supplementation and poststroke rehabilitation: a randomized, double-blind, placebo-controlled trial. Nutrients. (2019). 11:1295. doi: 10.3390/nu11061295

38. Holick MF. Vitamin D deficiency. N Engl J Med. (2007) 357:26681. doi: 10.1056/NEJMra070553

39. Yalbuzdag SA, Sarifakioglu B, Afsar SI, Celik C, Can A, Yegin T, et al. Is 25(OH)D Associated with cognitive impairment and functional improvement in stroke? A retrospective clinical study. J Stroke Cerebrovasc. (2015) 24:147986. doi: 10.1016/j.jstrokecerebrovasdis.2015.03.007

40. Turetsky A, Goddeau RP, Henninger N. Low serum vitamin $\mathrm{D}$ is independently associated with larger lesion volumes after ischemic stroke. J Stroke Cerebrovasc. (2015) 24:155563. doi: 10.1016/j.jstrokecerebrovasdis.2015.03.051

41. Huang $\mathrm{H}$, Zheng $\mathrm{T}$, Wang $\mathrm{S}$, Wei L, Wang $\mathrm{Q}$, Sun Z. Serum 25-hydroxyvitamin $\mathrm{D}$ predicts early recurrent stroke in ischemic stroke patients. Nutr Metab Cardiovasc Dis. (2016) 26:90814. doi: 10.1016/j.numecd.2016.06.009

42. Takeda M, Yamashita T, Sasaki N, Nakajima K, Kita T, Shinohara M, et al. Oral administration of an active form of vitamin D3 (calcitriol) decreases atherosclerosis in mice by inducing regulatory $\mathrm{T}$ cells and immature dendritic cells with tolerogenic functions. Arterioscler Thromb Vasc Biol. (2010) 30:2495-503. doi: 10.1161/ATVBAHA.110.215459

43. Won S, Sayeed I, Peterson BL, Wali B, Kahn JS, Stein DG. Vitamin D prevents hypoxia/reoxygenation-induced blood-brain barrier disruption via vitamin D receptor-mediated NF-kB signaling pathways. PLoS ONE. (2015) 10:e0122821. doi: 10.1371/journal.pone.0122821

44. Sinha A, Hollingsworth KG, Ball S, Cheetham T. Improving the vitamin D status of vitamin $\mathrm{D}$ deficient adults is associated with improved mitochondrial oxidative function in skeletal muscle. J Clin Endocr Metab. (2013) 98:E50913. doi: $10.1210 /$ jc. $2012-3592$ 
45. Annoni JM, Staub F, Bogousslavsky J, Brioschi A. Frequency, characterisation and therapies of fatigue after stroke. Neurol Sci. (2008) 29:S244-6. doi: 10.1007/s10072-008-0951-0

46. Choi-Kwon S, Choi J, Kwon SU, Kang DW, Kim JS. fluoxetine is not effective in the treatment of poststroke fatigue: a double-blind, placebo-controlled study. Cerebrovasc Dis. (2007) 23:103-8. doi: 10.1159/000097045

47. Vu LH, Whiteman DC, van der Pols JC, Kimlin MG, Neale RE. Serum vitamin D levels in office workers in a subtropical climate. Photochem Photobiol. (2011) 87:714-20. doi: 10.1111/j.1751-1097.2011.00899.x

Conflict of Interest: The authors declare that the research was conducted in the absence of any commercial or financial relationships that could be construed as a potential conflict of interest.
Publisher's Note: All claims expressed in this article are solely those of the authors and do not necessarily represent those of their affiliated organizations, or those of the publisher, the editors and the reviewers. Any product that may be evaluated in this article, or claim that may be made by its manufacturer, is not guaranteed or endorsed by the publisher.

Copyright (c) 2021 Wang, Zhao, Wang, Wu and Wang. This is an open-access article distributed under the terms of the Creative Commons Attribution License (CC BY). The use, distribution or reproduction in other forums is permitted, provided the original author(s) and the copyright owner(s) are credited and that the original publication in this journal is cited, in accordance with accepted academic practice. No use, distribution or reproduction is permitted which does not comply with these terms. 